\title{
Universal singular functions in local field theory
}

\author{
Alfred C. T. Wu \\ Department of Physics, University of Michigan, Ann Arbor, Michigan 48109 \\ (Received 11 Apri] 1977)
}

The singularity structure of the universal singular functions in local field theory is simply seen by the stationary-phase method applied to the Lorentz group manifold.

\section{INTRODUCTION}

The universal singular functions in local field theory were defined about twenty years ago. ${ }^{1,2}$ One assumes that it is important to know the set of vacuum expectation values of products of local field operators, in the sense that knowing all the moments of a field essentially characterizes the field itself. ${ }^{3}$ In the Fourier analysis of these Wightman functions, a covariant decomposition of the momentum-space-support permits a group theoretic extraction of the "angular" part from the volume integral while leaving behind the "radial" (or scalar product) part where the dynamics enters.

The extraction of the universal singular functions is done as follows. ${ }^{2}$ Consider the $(n+1)$-fold product as a function of $n 4$-vectors,

$$
\begin{aligned}
F^{(n)}\left(\zeta_{k}\right) \equiv & \left\langle 0\left|A_{1}\left(x_{1}\right) \cdots A_{n+1}\left(x_{n+1}\right)\right| 0\right\rangle, \quad \begin{array}{l}
\zeta_{k}=x_{k+1}-x_{k} \\
k=1, \ldots, n
\end{array} \\
= & \text { const } \cdot \int \Pi d p_{k} \exp \left(-i \sum p_{k} \cdot \zeta_{k}\right) G^{(n)}\left(p_{1}, \ldots, p_{n}\right) \\
= & \text { const } \cdot \int \Pi d\left(\Lambda p_{k}\right) \exp \left[-i \sum\left(\Lambda p_{k}\right) \cdot \zeta_{k}\right] \\
& \times G^{(n)}\left(\Lambda p_{1}, \ldots, \Lambda p_{n}\right) \\
= & \text { const } \cdot \int d \nu(\omega) \widetilde{G}^{(n)}(\omega) \Delta(M)
\end{aligned}
$$

In going from (2) to (3), we emphasize the invariance under $p \rightarrow \Lambda p ; \Lambda$ is an element of the homogeneous proper orthochronous Lorentz group. The universal singular function $\Delta(M)$ is simply the properly written covariant expression of the "angular" integral of the Fourier exponential factors, namely

$$
\Delta(M)=\int d \mu(\Lambda) \exp [-i \operatorname{tr}(\Lambda M)]
$$

where $d \mu(\Lambda)$ denotes the Haar measure on the Lorentz group, and $M$ is a matrix whose elements are formed by the mixed products of $p$ and $\zeta$,

$$
M_{\nu}^{\mu}=\sum_{k=1}^{n} p_{k}{ }^{\mu} \zeta_{k \nu}
$$

The formalism can be set up for Lorentz spaces of arbitrary $(m+1)$ space-time dimensions where $M$ and $\Lambda$ are $(m+1) \times(m+1)$ matrices. For the cases $n \leqslant m$, there is a certain economy in studying $F^{(n)}$ in a $(n+1)$ dimensional space-time, the nontrivial cases are therefore those for which the number of independent vectors equals the rank of the matrices, i. e., $n=m+1$.

Analytic structure and explicit evaluation of $\Delta(M)$ would be items of obvious interest. Let us first summarize what is known on these.

(a) Explicit forms of $\Delta(M)$ are known only for some special cases, namely $n=1,2$ for $m=3^{1,2}$ and $n \leqslant 3$ for $m=1 .^{2}$ There have been attempts to evaluate the integrals for higher $n$ and $m$, but the results are by no means complete. ${ }^{4}$

(b) The analytic structure of $\Delta(M)$ of course can be read off in cases where the singularities of the integrand can be suitably displayed. The Lorent $z$ group manifold technique (4) is an alternate (and hopefully more transparent) approach to the analyticity of the vacuum expectation values. The other approach is the so-called generalized singular functions $\Delta_{n+1}^{+} \cdot{ }^{5-9}$ Of course, the $\Delta(M)$ function and the $\Delta_{n+1}^{+}(z ; a)$ function are closely related, but the spirit and the techniques involved are sufficiently different to warrant a separate analysis of $\Delta(M)$. The main result on the $\Delta_{n+1}^{+}(z ; a)$ functions is that their singularity domains are given by the following trace manifold $d^{5-9}$

$$
\sum_{k=1}^{n}\left( \pm \sqrt{\tau_{k}}\right)=\text { real, }
$$

where $\tau_{k}$ 's are the eigenvalues of the product matrix $Z a, Z$ and $a$ are the Gram matrices in the $x$ space and $p$ space respectively,

$$
Z_{i j}=\zeta_{i} \circ \zeta_{j}, \quad a_{i j}=p_{i} \circ p_{j} 。
$$

The present work is prompted by the desire of finding a simpler way to see the sources of singularities of $\Delta(M)$, namely the manifold

$$
\operatorname{Im}\left\{\operatorname{tr}\left[ \pm(\tilde{M} M)^{1 / 2}\right]\right\}=0 .
$$

$\tilde{M}$ is the transpose with the built in metric,

$$
(\tilde{M})^{\mu}{ }_{v} \equiv M_{\nu}{ }^{\mu} \text {. }
$$

In matrix notation, we have

$$
\tilde{M}=G M^{T} G \text {, }
$$

where $M^{T}$ denotes the ordinary matrix transpose and $G$ is the matrix of $g_{\mu_{\nu}}\left(g_{00}=1, g_{i i}=-1\right.$, and zero otherwise). We note in passing that the result (9) stated above for $\Delta(M)$ is consistent with the result (7) on $\Delta_{n+1}^{+}$ by virtue of the following identity,

$$
\operatorname{tr}(\tilde{M} M)=\operatorname{tr}(Z a) \text { 。 }
$$

The method of stationary phase is used here to establish the following relation,

$$
\operatorname{tr}(\Lambda M)_{\substack{\text { stationary } \\ \text { phase }}}=\operatorname{tr}\left[ \pm(\tilde{M} M)^{1 / 2}\right] .
$$

In this way, the singularity source of Eq. (9) is viewed as that coming from the ceasing of the exponential damping subject to the stationary phase prescription. The present analysis shows that result (9) can be established directly from (5) in a reasonably transparent manner. A simple way of visualizing result (12) is that 
the right-hand side of (12) is actually a minimum as a consequence of the extremum principle. This minimum can perhaps be seen by invoking the Schwarz inequality. ${ }^{10}$

The motivation for the stationary phase method is briefly discussed in Sec. II. For the sake of readability, the parametrization of the Lorentz transformation $\Lambda$ is stated in Sec. III. The two-dimensional case is reviewed in Sec. IV where the result of the stationary phase method is compared with that obtained by the explicit evaluation. In Sec. V, higher rank cases are discussed with the aid of the diagonalization procedure on the matrix $M$.

\section{MOTIVATION FOR THE STATIONARY PHASE METHOD}

The method of stationary phase $\mathrm{e}^{11}$ is used here as a device of handling oscillatory exponential terms. Technically, the applicability of the method requires a limiting procedure such as a large parameter which enhances the oscillation. This may be understood by an appeal to the classical path integral by recovering a $\hbar^{-1}$ factor in the exponential and taking the limit $\hbar-0$. A similar approach to the Feynman integral was discussed by Nakanishi. ${ }^{12}$ Application to the $\Delta_{n+1}^{+}$functions was studied by Fäldt. ${ }^{9}$

\section{LORENTZ GROUP MANIFOLD: PARAMETRIZATION OF $\Lambda$ AND $d \mu(\Lambda)$}

For a given space-time dimension $m+1$, the parametrization of the Lorentz transformation $\Lambda$ and the invariant volume element $d \mu(\Lambda)$ can be worked out by standard procedure. One convenient scheme of parametrizing a general Lorentz transformation $\Lambda$ is to decompose it into product form of a pure boost sandwiched between appropriate space rotations. ${ }^{13} \mathrm{As}$ is well known, this is a judicious generalization of the Euler angle decomposition for the rotation in 3-space. Explicitly, we have

(a) $m=1$ (1-space, 1-time)

$$
\begin{aligned}
& \Lambda=\left(\begin{array}{ll}
\cosh \chi & \sinh \chi \\
\sinh \chi & \cosh \chi
\end{array}\right) \equiv \Lambda_{01}, \\
& d^{(2)} \mu(\Lambda)=d \chi, \quad-\infty<\chi<\infty ;
\end{aligned}
$$

(b) $m=2(2$-space, 1 -time)

$$
\begin{aligned}
& \Lambda=R_{12}(\theta) \Lambda_{01}(\chi) R_{12}(\phi) \\
& R_{12}=\left(\begin{array}{ccc}
1 & 0 & 0 \\
0 & \cos \theta & \sin \theta \\
0 & -\sin \theta & \cos \theta
\end{array}\right), \\
& \Lambda_{01}(\chi)=\left(\begin{array}{ccc}
\cosh \chi & \sinh \chi & 0 \\
\sinh \chi & \cosh \chi & 0 \\
0 & 0 & 1
\end{array}\right), \\
& d^{(3)} \mu(\Lambda)=\sinh \chi d \chi d \theta d \phi, \quad 0 \leqslant \theta, \quad \phi \leqslant 2 \pi,-\infty<\chi<\infty ;
\end{aligned}
$$

(c) $m=3(3$-space, 1 -time)

$$
\Lambda=R_{23}(\theta) R_{13}(\psi) R_{23}(\phi) \Lambda_{01}(\chi) R_{13}(\alpha) R_{23}(\beta),
$$

where $R_{i j}(\theta)$ denotes a rotation in the $\left(x^{i}, x^{j}\right)$ plane, e.g. ,

$$
R_{23}(\theta)=\left(\begin{array}{cccc}
1 & 0 & 0 & 0 \\
0 & 1 & 0 & 0 \\
0 & 0 & \cos \theta & \sin \theta \\
0 & 0 & -\sin \theta & \cos \theta
\end{array}\right),
$$

$\Lambda_{01}(\chi)$ denotes a boost [like (14c)] except here it is $4 \times 4$, and

$$
\begin{aligned}
& d^{(4)} \mu(\Lambda)=\sinh ^{2} \chi d \chi \sin \alpha d \alpha \sin \psi d \psi d \theta d \phi d \beta, \\
& 0<\chi<\infty, \quad 0 \leqslant \alpha, \psi \leqslant \pi, \quad 0 \leqslant \theta, \beta, \phi \leqslant 2 \pi .
\end{aligned}
$$

(d) For general $(m+1)$-space-time, we may write

$$
\Lambda=R_{1} \Lambda_{01} R_{2},
$$

where $R_{1}$ denotes a rotation in $m$-space, $R_{2}$ would be a rotation in $m$-space also except for two reasons. One reason is that this would yield $\frac{1}{2}(m-1)(m-2)$ excessive parameters. The other reason is that the $\Lambda_{01}$ matrix has a $(m-1) \times(m-1)$ identity submatrix in it which permits a tunnelling of a rotation in $(m-1)$-space. These two situations can be reconciled if $R_{2}$ is the quotient of $R_{m} / R_{m-1}$, which yields $(m-1)$ parameters. [Cf. Eq. (15a).] The volume element may be written as

$$
d^{(m+1)} \mu(\Lambda)=\sinh ^{m-1} \chi d \chi d \mu\left(R_{m}\right) d \mu\left(R_{m} / R_{m-1}\right) .
$$

In the next section, we study the simplest case $m=1$, $n=2$.

\section{A TWO-DIMENSIONAL EXAMPLE REVISITED: EXPLICIT EVALUATION VERSUS STATIONARY PHASE PRESCRIPTION}

For the sake of illustration, consider the following example $m=1, n=2$, namely, the case of a three-point function in two-dimensional space-time.

\section{A. Explicit evaluation}

Write

$$
M=\left(\begin{array}{ll}
M_{0}^{0} & M_{1}^{0} \\
M_{0}^{1} & M_{1}^{1}
\end{array}\right), \quad \sigma_{1}=\left(\begin{array}{ll}
0 & 1 \\
1 & 0
\end{array}\right),
$$

with $\Lambda$ given by (13a), we have

$$
\begin{aligned}
\operatorname{tr}(\Lambda M) & =(\operatorname{tr} M) \cosh \chi+\operatorname{tr}\left(M \sigma_{1}\right) \sinh \chi \\
& =\left[(\operatorname{tr} M)^{2}-\left(\operatorname{tr}\left(M \sigma_{1}\right)\right)^{2}\right]^{1 / 2} \cosh \left(\chi+\chi_{0}\right) \\
& =[\operatorname{tr}(\tilde{M} M)+2 \operatorname{det} M]^{1 / 2} \cosh \left(\chi+\chi_{0}\right) \\
& =\operatorname{tr}\left[ \pm(\tilde{M} M)^{1 / 2}\right] \cosh \left(\chi+\chi_{0}\right),
\end{aligned}
$$

where

$$
\tanh \chi_{0}=\operatorname{tr}\left(M \sigma_{1}\right) / \operatorname{tr} M \text { 。 }
$$

The Lorentz transpose $\tilde{M}$ was defined in (10). In the last step leading to (19), use has been made of the trace identity for a rank two matrix, namely

$$
\operatorname{tr} A^{2}-(\operatorname{tr} A)^{2}+2 \operatorname{det} A=0,
$$

with $A=(\tilde{M} M)^{1 / 2}$.

In this case, evaluation of $\Delta(M)$ readily gives

$$
\begin{aligned}
\Delta_{2}^{(3)}(M) & =\int_{-\infty}^{\infty} d \chi\left\{\exp i\left(\operatorname{tr}\left[ \pm(\tilde{M} M)^{1 / 2}\right]\right)\right\} \cosh \left(\chi+\chi_{0}\right) \\
& =i \pi H_{0}^{(1)}\left(\operatorname{tr}\left[ \pm(\tilde{M} M)^{1 / 2}\right]\right) .
\end{aligned}
$$


From (22) it is seen that the source of singularities is at those points where there is no damping for the Hankel function, and that is where the argument for the Hankel function becomes real and positive, which lies on the manifold (9).

\section{B. Stationary phase prescription}

We indicate here how the stationary phase method can be made to give the desired result. The zeros of $(\partial / \partial \chi) \operatorname{tr}(\Lambda M)$ may be found from (18) or (19). The stationary phase prescription gives

$$
\chi=-\chi_{0}
$$

and

$$
\left.\operatorname{tr}(\Lambda M)\right|_{\mathrm{SP}}=\operatorname{tr}\left[ \pm(\tilde{M} M)^{1 / 2}\right],
$$

verifying (12) for this simple example.

It is obvious that as $(n, m)$ goes up, the control of the integration scheme becomes increasingly difficult. On the other hand, the stationary phase prescription which is easier to handle, hopefully will give the desired result on the singularity structure.

We note in passing that on account of the extreme simplicity of the above example, there is no need to invoke the diagonalization of the $M$ matrix in (18). However, the diagonalization technique will become highly desirable in the treatment of higher rank cases.

\section{HIGHER RANK CASES}

\section{A. Two lemmas on diagonalization}

To facilitate algebraic manipulations, it will be convenient to utilize two lemmas on matrix diagonalization. The first is due to Hall, ${ }^{14}$ and the second one is an obviously parallel statement.

Lemma $1\left(\right.$ Hall $\left.^{14}\right)$ : A $2 \times 2$ matrix $M, M^{\mu}{ }_{\nu}=\sum_{k=1}^{2} p_{k}{ }^{\mu} \eta_{k \nu}$, with positive timelike $p_{k}, \eta_{k}$ can be diagonalized by two boosts

$$
M^{\prime}=\Lambda\left(\chi_{1}\right) M \Lambda\left(\chi_{2}\right),
$$

where $\Lambda$ is of the form (13a).

Proof: We simply exhibit the angles that will render $M^{\prime}$ diagonal. They are

$$
\begin{aligned}
& \tanh 2 \chi_{1}=-\operatorname{tr}\left(M i \sigma_{2} M\right) / \operatorname{tr}\left(\tilde{M} \sigma_{3} M\right), \\
& \tanh 2 \chi_{2}=\operatorname{tr}\left(M i \sigma_{2} \tilde{M}\right) / \operatorname{tr}\left(M \sigma_{3} \tilde{M}\right),
\end{aligned}
$$

where

$$
i \sigma_{2}=\left(\begin{array}{cc}
0 & 1 \\
-1 & 0
\end{array}\right), \quad \sigma_{3}=\left(\begin{array}{cc}
1 & 0 \\
0 & -1
\end{array}\right)
$$

Remark: The timelike condition on $p_{k}$ and $\eta_{k}$ guarantees that such real $\Lambda\left(\chi_{1}\right)$ and $\Lambda\left(\chi_{2}\right)$ of the form (13a) exist. The usefulness of this lemma lies in the invariance of the volume element and the integrand in (5) under (25). In applying this lemma to the configuration space difference vectors lying in the tube domain, ${ }^{15}$ it suffices to take $\xi_{k}=-i \eta_{k}$ with $\eta_{k}$ positive timelike and to continue analytically from there.

Lemma 2: A $2 \times 2$ matrix $N$ can be diagonalized by two rotations $[N$ is understood to consist of spatial indices only]

$$
N^{\prime}=R\left(\theta_{1}\right) N R\left(\theta_{2}\right),
$$

where $R(\theta)$ is of the form

$$
\left(\begin{array}{cc}
\cos \theta & -\sin \theta \\
\sin \theta & \cos \theta
\end{array}\right)
$$

Proof: The diagonalization is achieved with the angles $\theta_{1}$ and $\theta_{2}$ given by

$$
\begin{aligned}
& \tan 2 \theta_{1}=-\operatorname{tr}\left(N^{T} \sigma_{1} N\right) / \operatorname{tr}\left(N^{T} \sigma_{3} N\right), \\
& \tan 2 \theta_{2}=\operatorname{tr}\left(N \sigma_{1} N^{T}\right) / \operatorname{tr}\left(N \sigma_{3} N^{T}\right) .
\end{aligned}
$$

Remark: Actually, the spatial part $N$ of the matrix $M$ being a symmetric submatrix can be diagonalized by orthogonal similarity transformations (spatial rotations). Then Lemma 1 can be repeatedly applied in the $(0 k)$ planes to remove the nondiagonal elements in the first row and the first column. It should be realized that prior spatial diagonalization is essential because of the noncommutativity of boosts $\Lambda_{0 i}$ and $\Lambda_{0 j}$ in the different directions.

\section{B. Successive rotations and boosts}

As stated in Sec.III, a general Lorentz transformation in the $(m+1)$-dimensional space-time may be parametrized as a product of a pure boost [say in the (01) plane] sandwiched between two sets of spatial rotations. The latter may be decomposed into suitable products of plane rotations. We may utilize the rotational freedom to effect first the block diagonalization of $M$ in the spatial indices in accordance with the remark following Lemma 2. Thus

$$
\begin{aligned}
\operatorname{tr}(\Lambda M) & =\operatorname{tr}\left(R_{1} \Lambda_{01} R_{2} M\right)=\operatorname{tr}\left(\Lambda_{01} R_{2} M R_{1}\right) \\
& =\operatorname{tr}\left(\Lambda_{01} \hat{R}_{2} \hat{M} \hat{R}_{1}\right)=\operatorname{tr}\left(\hat{R}_{1} \Lambda_{01} \hat{R}_{2} \hat{M}\right) \\
& =\operatorname{tr}(\hat{\Lambda} \hat{M}),
\end{aligned}
$$

where

$$
\hat{M}=R_{2}^{\prime} M R_{1}^{\prime}
$$

is diagonal in the spatial indices

$$
\begin{aligned}
& \hat{R}_{2}=R_{2} R_{2}^{\prime-1}, \quad \hat{R}_{1}=R_{1}^{\prime-1} R_{1}, \\
& \hat{\Lambda}=\hat{R}_{1} \Lambda_{01} \hat{R}_{2} .
\end{aligned}
$$

What remains to be done is to use Hall's lemma repeatedly to render $\hat{M}$ diagoanl in the time components also.

We conclude that without loss of generality the $M$ matrix in the integrand of (5) can be suitably diagonalized.

\section{Stationariness of the trace manifold}

Suitable parametrization of the Lorentz transformation and the proper diagonalization of the matrix $M$ are thus two technical devices which considerably simplify the evaluation of the trace quantity. We have (the matrix $M$ below is understood to be diagonal)

$$
\begin{aligned}
\operatorname{tr}(\Lambda M) & =\operatorname{tr}\left(\Lambda_{01} R M S\right), \quad R, S \text { are rotations } \\
& =\left(M_{0}^{0}+R^{1}{ }_{k} M^{k}{ }_{j} S_{1}^{j}\right) \cosh \chi+\operatorname{tr}(\overline{\bar{R}} \overline{\bar{M}} \overline{\bar{S}}),
\end{aligned}
$$


where the double bar quantities denote the corresponding $(m-2) \times(m-2)$ submatrices with indices running from 2 to $m$. The $\sinh \chi$ term is absent on account of the accomplished diagonalization of $M$. The demand of the stationary phase in each of the angle variable (Lorentz as well as Euclidean) then results in the following. First the stationariness in the boost angle $\chi$ which implies $\chi=0$ gives

$$
\left.\operatorname{tr}(\Lambda M)\right|_{\text {stat。 boost }}=M_{0}^{0}+\operatorname{tr}\left(\bar{R}^{\prime} \bar{M}\right),
$$

where the single bar quantities denote the corresponding $(m-1) \times(m-1)$ submatrices with the indices running from 1 to $m$, and $\bar{R}^{\prime}=\bar{S} \bar{R}$. For the remaining spatial indices, it is obvious that the orthogonal manifold for the rotational matrices would simply reduce the trace quantity in (30) to assume the value $\operatorname{tr}\left[ \pm\left(\bar{M}^{T} \bar{M}\right)^{1 / 2}\right]$. Hence

$$
\left.\operatorname{tr}(\Lambda M)\right|_{\mathrm{SP}}=\operatorname{tr}\left[ \pm(\tilde{M} M)^{1 / 2}\right]
$$

which is the result stated in (12).

\section{ACKNOWLEDGMENT}

The author is indebted to Professor A.S. Wightman for introducing him to the subject matter.
${ }^{1}$ A. S. Wightman and D. Hall, Phys. Rev. 99, 674 (1955). ${ }^{2}$ D. Hall, thesis, Princeton University, 1956, unpublished; esp. Chaps. 4-6.

${ }^{3}$ A.S. Wightman, Phys. Rev. 101, 860 (1956); R. Jost, The General Theory of Quantum Fields (Am. Math. Soc., Providence, R.I., 1965).

${ }^{4}$ E.g. , A.S. Wightman (private communication, 1962).

${ }^{5}$ G. Källén and A.S. Wightman, K. Dan. Vidensk. Selsk. Mat. Fys. Skr. 1, No. 6 (1958).

${ }^{6} \mathrm{G}$. Källén and H. Wilhelmsson, K. Dan. Videns. Selsk. Mat. Fys. Skr. 1, No. 9 (1958).

${ }^{7} \mathrm{G}$. Källén, Lecture Notes (Les Houches, 1960), in Relation de dispersion et particules élémentaires (Hermann, Paris, 1960), pp. 229-313.

${ }^{8}$ For completeness, other work by Källen's students on the $\Delta^{+}$functions may be cited here: I. Nieminen, Nucl. Phys. $37,250(1962)$ studied the $\Delta_{4}^{+}$in lower dimensional spaces; G. Fäldt, ibid. 59, 449 (1964) discussed the $\Delta_{n^{+1}}^{+}$in $n$-dimensional space-time.

${ }^{9}$ G. Fäldt, Nucl. Phys. B 59, 449 (1964).

${ }^{10}$ The author is indebted to Prof. L. Michel for a remark on this.

${ }^{11}$ See, e.g., H. Jeffreys and B.S. Jeffreys, Methods of Mathematical Physics (Cambridge U. P., Cambridge, 1956), 3rd ed., esp. p. 506.

${ }^{12}$ N. Nakanishi, Prog. Theor. Phys. 39, 768 (1968).

${ }^{13}$ See, e.g., A.s. Wightman, Lecture Notes (Les Houches, 1960), volume cited in Ref. 7, esp., p. 167.

${ }^{14}$ D. Hall, Ref. 2 , pp. VI. 3

${ }^{15}$ A.S. Wightman, Proc. Indian Math. Soc. 24, 625 (1960). 